\title{
EXTREMAL PARTITIONS AND DISTORTION UNDER THE MONTEL BOUNDED UNIVALENT MAPS
}

\author{
ALEXANDER VASIL'EV \\ (Received 12 October 2000; revised 8 October 2001) \\ Communicated by P. C. Fenton
}

\begin{abstract}
Extremal partitions of domains into configurations of certain topological form are studied. The extremal value of the weighted sum of reduced moduli of circular domains and digons is obtained. These results are applied to some problems about distortion under bounded conformal maps of the unit disk with two preassigned values.
\end{abstract}

2000 Mathematics subject classification: primary 30C75; secondary $30 \mathrm{C} 45$.

Keywords and phrases: moduli method, extremal problem, univalent function, Montel function, distortion.

\section{Introduction}

We start with the problem of extremal partition associated with certain quadratic differentials in the unit disk. These differentials are allowed to have poles of second order. The problem of finding the extremal value of a weighted sum of the reduced moduli of simply connected domains of the partition is studied in Section 2 . We also are concerned with the reducedmoduli of digons. Then we apply these results to the problems about distortion under bounded conformal maps of the unit disk with two preassigned values.

Denote by $U(M)$ the disk $\{z:|z|<M\}$ in the complex plane $\mathbb{C}, U(1)=: U$. We consider the class $\mathscr{M}^{M}(\omega)$ of all univalent holomorphic maps $f: U \rightarrow U(M)$ (with $M>1$ ) normalized by conditions $f(0)=0$ and $f(\omega)=\omega$, where $\omega \in(0,1)$. Among complex analysts such a kind of normalization is known as the Montel normalization. Denote by $\mathscr{M}(\omega):=\mathscr{M}^{\infty}(\omega)$. Krzyż, Złotkiewicz and Libera contributed to the 
studies of these functions [4-7, 12]. In particular, Krzyż and Zlotkiewicz [7] have found the Koebe set for the class $\mathscr{M}(\omega)$, that is $\bigcap_{f \in \mathscr{N}(\omega)} f(U)$. Krzyż [4-6] has defined the set of values of $f(z)$, for $z$ fixed in $U$, by the variational method. This study was continued by Reade and Zlotkiewicz [12]. The bounded Montel function has been studied by Libera and Zlotkiewicz [10], and by the author and Pronin [14-17]. In [10] the sharp estimates of $\left|f^{\prime}(0)\right|$, the upper bound of $|f(z)|$, and the non-sharp boundary of the Koebe set was obtained. Then, we got the sharp form of the Koebe set for this class [16], making use of the extremal length method.

We define two canonical functions that play the role of the Koebe function in the class $\mathscr{M}^{M}(\omega)$

$$
K_{1}(z)=M k_{0}^{-1}\left(M\left(\frac{1+\omega}{M+\omega}\right)^{2} k_{0}(z)\right), \quad K_{2}(z)=M k_{\pi}^{-1}\left(M\left(\frac{1-\omega}{M-\omega}\right)^{2} k_{\pi}(z)\right),
$$

where $k_{\theta}$ is the usual Koebe function $k_{\theta}(z)=z\left(1+z e^{i \theta}\right)^{-2}$.

From [10] it follows that if $f \in \mathscr{M}^{M}(\omega)$, then

$$
\frac{M^{2}(1-\omega)^{2}}{(M-\omega)^{2}} \leq\left|f^{\prime}(0)\right| \leq \frac{M^{2}(1+\omega)^{2}}{(M+\omega)^{2}} .
$$

The equality sign is attained by the function $K_{1}$ for the right-hand inequality and by the function $K_{2}$ for the left-hand inequality. It is not difficult to obtain the analogous estimates for $\left|f^{\prime}(\omega)\right|$ :

$$
\frac{(M+\omega)(1-\omega)}{(M-\omega)(1+\omega)} \leq\left|f^{\prime}(\omega)\right| \leq \frac{(M-\omega)(1+\omega)}{(M+\omega)(1-\omega)},
$$

with the same extremal functions. Thus, the range $\mathfrak{M}_{f}$ of the system of functionals $\left(\left|f^{\prime}(0)\right|,\left|f^{\prime}(\omega)\right|\right)$ lies within the rectangle defined by the inequalities (1) and (2). In the class $\mathscr{M}(\omega)$ this problem has been considered in [1]. Section 3, following the preliminaries in Section 2, is devoted to description of the sharp boundary curve of this range.

\section{Extremal partition by circular domains and digons}

2.1. Let $D$ be a hyperbolic simply connected domain and $z_{0} \in D$. Denote by $m\left(D, z_{0}\right)$ the usual reduced modulus of the domain $D$ with respect to the point $z_{0}$. The domain $D$ with its fixed point $z_{0}$ is called a circular domain. If $R\left(D, z_{0}\right)$ is the conformal radius of $D$ with respect to $z_{0}$, then $m\left(D, z_{0}\right)=(1 / 2 \pi) \log R\left(D, z_{0}\right)$ if $\left|z_{0}\right|<\infty$ and $m(D, \infty)=-(1 / 2 \pi) \log R(D, \infty)$ otherwise. The reduced modulus is changed under a conformal map $f$ by the rule $m\left(f(D), f\left(z_{0}\right)\right)=m\left(D, z_{0}\right)+$ $(1 / 2 \pi) \log \left|f^{\prime}\left(z_{0}\right)\right|$ when $\left|f\left(z_{0}\right)\right|<\infty$. If $f(z)=a_{-1} /\left(z-z_{0}\right)+a_{0}+a_{1}\left(z-z_{0}\right)+\cdots$, then $m(f(D), \infty)=m\left(D, z_{0}\right)-(1 / 2 \pi) \log \left|a_{-1}\right|$. 
2.2. We start with the problem of the extremal partition of the complex plane. Let $S_{0}=\mathbb{C} \backslash\{0,1\}$ be the punctured Riemann sphere. We consider on $S_{0}$ a pair $\left(\gamma_{1}, \gamma_{2}\right)$ of curves, where $\gamma_{1}=\{w:|w|=1 / \varepsilon\}$ and $\gamma_{2}=\{w:|w|=\varepsilon\}$. Here $\varepsilon$ is sufficiently small and such that 1 belongs to the doubly connected domain between $\gamma_{1}$ and $\gamma_{2}$ on $\mathbb{C}$. Let $\mathfrak{D}$ be the set of all pairs $\left(D_{1}, D_{2}\right)$ consisting of circular domains of homotopy type $\left(\gamma_{1}, \gamma_{2}\right)$. This means that the boundary $\partial D_{j}$ is freely homotopic to $\gamma_{j}$ on $S_{0}$. Then the problem of extremal partition of $S_{0}$ reduces to the problem of finding the maximum of the sum

$$
\alpha_{1}^{2} m\left(D_{1}, \infty\right)+\alpha_{2}^{2} m\left(D_{2}, 0\right),
$$

where $\left(D_{1}, D_{2}\right) \in \mathfrak{D}$. Without loss of generality assume $\alpha_{1}=1, \alpha_{2}=\alpha, \alpha \in[0, \infty)$ and denote by $M(\alpha)$ the maximum of the sum in (3). From [3,8] it follows that there is a unique pair $\left(D_{1}^{*}, D_{2}^{*}\right)$ which is extremal for this problem. $D_{1}^{*}$ and $D_{2}^{*}$ are the circular domains in the trajectory structure of the differential

$$
Q(z) d z^{2}=-A \frac{(z-c) d z^{2}}{z^{2}(z-1)}, \quad A>0 .
$$

Here $A$ and $c$ are some functions with respect to $\alpha$. If $\alpha=1$, then the lengths of trajectories from $D_{1}^{*}$ and $D_{2}^{*}$ are equal and $c(1)=1$. In this case $M(1)=0$, $D_{1}^{*}=\{z:|z|>1\}$ and $D_{2}^{*}=U$. If $\alpha=0$ or $\alpha \rightarrow \infty$, then the domains $D_{2}^{*}$ and $D_{1}^{*}$ respectively degenerate and in both cases $M(0)=M(\infty)=(1 / 2 \pi) \log 4$. In other cases we have the following theorem.

THEOREM 2.1. Let $0<\alpha<\infty, \alpha \neq 1$. Then

$$
\begin{aligned}
& m\left(D_{1}^{*}, \infty\right)=\frac{1}{2 \pi} \log \frac{4|1-\alpha|^{\alpha-1}}{|1+\alpha|^{\alpha+1}}, \\
& m\left(D_{2}^{*}, 0\right)=\frac{1}{2 \pi} \log \frac{4 \alpha^{2}|1-\alpha|^{(1 / \alpha)-1}}{|1+\alpha|^{(1 / \alpha)+1}} .
\end{aligned}
$$

Moreover, for the differential $Q(z) d z^{2}$, we calculate $A=1 / 4 \pi^{2}$ and $c=\alpha^{2}$.

Proof. We consider the conformal map $u=u(z)$ whose inverse is

$$
z=(c-1) \frac{1-\cos u}{(1-c)-(1+c) \cos u}+1 .
$$

Consequently, we obtain the representation of the differential $\varphi$ in terms of the parameter $u$ in regular points

$$
Q(z) d z^{2}=Q_{1}(u) d u^{2}=-\frac{A(c-1)^{2} c(1+\cos u)^{2}}{((1-c)-(1+c) \cos u)^{2} \cos ^{2} u} d u^{2} .
$$


We study the trajectory structure of the above quadratic differential. The differential $Q_{1}(u) d u^{2}$ has zeros of order 4 at the points $\pi+2 \pi k$ which are the images of $c$ under the map $u(z)$. Then, $u(0)=\pi / 2+k \pi$ and $u(\infty)=\theta_{k}=\cos ^{-1}(1-c) /(1+c)$ are the poles of the second order. The points $u(1)=2 \pi k$ are regular for this differential.

Let us consider a fixed branch of the function $u(z)$ which maps $\overline{\mathbb{C}} \backslash[c, 1]$ onto a strip $0<\operatorname{Re} u<\pi$. For $\alpha<1$, the circular domain $D_{2}^{u}=u\left(D_{2}^{*}\right)$ is bounded by a critical trajectory of $Q_{1}(u) d u^{2}$ that starts and ends at $\pi$, so that $\pi / 2 \in D_{2}^{u}$, and $\theta_{1} \notin D_{2}^{\mu}\left(\theta_{1} \in(0, \pi / 2)\right)$. The circular domain $D_{1}^{\mu}=u\left(D_{1}^{*}\right)$ is bounded by the same trajectory and the straight lines $\operatorname{Re} u=0, \pi$. For $\alpha>1$, the boundary of the domain $D_{1}^{u}$ is the critical trajectory of $Q_{1}(u) d u^{2}$ that starts and ends at $\pi$, so that $\theta_{1} \in D_{1}^{u}$, $\theta_{1} \in(\pi / 2, \pi)$. The circular domain $D_{2}^{u}=u\left(D_{2}^{*}\right)$ is bounded by the same trajectory and the straight lines $\operatorname{Re} u=0, \pi$.

Let $\zeta_{j}(u), j=0,1$ be univalent conformal maps from domains $D_{j}^{u}$ onto the unit disk $U$ such that $\zeta_{1}(\pi / 2)=\zeta_{2}\left(\theta_{1}\right)=0$ in the case $\alpha<1$ or $\zeta_{2}(\pi / 2)=\zeta_{1}\left(\theta_{1}\right)=0$ in the case $\alpha>1$. These functions satisfy, in the domains $D_{j}^{\mu}$, the differential equations

$$
\alpha_{j} \frac{d \zeta_{j}(u)}{\zeta_{j}(u)}=2 \pi \sqrt{-Q_{1}(u)} d u, \quad \alpha_{1}=1, \alpha_{2}=\alpha
$$

or in terms of the parameter $z$

$$
\alpha_{j}^{2}\left(\frac{d \zeta_{j}(u(z))}{\zeta_{j}(u(z))}\right)^{2}=-4 \pi^{2} Q(z) d z^{2} .
$$

Taking a limit in (9) either as $z \rightarrow \infty$ (if $j=1$ ) or as $z \rightarrow 0$ (if $j=2$ ), we obtain $A=1 / 4 \pi^{2}$ and $c=\alpha^{2}$.

To calculate the reduced moduli we consider foremost the case $\alpha<1$. The part $[\pi / 2+\delta, \pi]$ of the orthogonal trajectory of the differential $Q(u) d u^{2}$ for a sufficiently small $\delta$ has a preimage $\left[\varepsilon_{1}, c\right]$ under the map $u(z)$. From (6) we derive

$$
\delta=\frac{1-c}{2 c} \varepsilon_{1}+O\left(\varepsilon_{1}^{2}\right) .
$$

The segment $[\pi / 2+\delta, \pi]$ has an image $[\varepsilon, 1]$ under the map $\zeta=\zeta_{2}(u)$.

Let $z=f_{2}(\zeta)=A_{1} \zeta+A_{2} \zeta^{2}+\cdots$ be a function from $U$ onto $D_{2}^{*}$. Then the conformal radius of $D_{2}^{*}$ can be calculated as $R\left(D_{2}^{*}, 0\right)=1 /\left|A_{1}\right|$. Using (7) we have

$$
\begin{aligned}
\sqrt{-Q_{1}(u)}= & \pm \frac{\alpha}{2 \pi} \frac{d}{d u}\left(\frac{1}{\tan \left(\theta_{1} / 2\right)} \log \left[ \pm\left(\frac{\tan (u / 2)-\tan \left(\theta_{1} / 2\right)}{\tan (u / 2)+\tan \left(\theta_{1} / 2\right)}\right)\right]\right. \\
& \left.+\frac{1}{2} \log \frac{1+\sin u}{1-\sin u}\right),
\end{aligned}
$$

where we choose after the logarithm $(+)$ if $\left(\tan (u / 2)-\tan \left(\theta_{1} / 2\right)\right) /(\tan (u / 2)+$ $\left.\tan \left(\theta_{1} / 2\right)\right)>0$ and $(-)$ otherwise. Moreover, $\tan \left(\theta_{1} / 2\right)=\sqrt{c}=\alpha$. We fix a 
branch of the root in the left-hand side of (11) choosing (-) after the equality sign. Integrating (8) along relevant segments $(j=2)$ we obtain

$$
\varepsilon=\left(\frac{\tan (\pi / 4+\delta / 2)+\alpha}{\tan (\pi / 4+\delta / 2)-\alpha}\right)^{1 / \alpha}\left(\frac{1-\cos \delta}{1+\cos \delta}\right)^{1 / 2}=\left(\frac{1+\alpha}{1-\alpha}\right)^{1 / \alpha} \frac{\delta}{2}+O\left(\delta^{2}\right),
$$

and finally using (10) we have

$$
A_{1}=\frac{4 c}{1-c}\left(\frac{1-\alpha}{1+\alpha}\right)^{1 / \alpha}
$$

As $\alpha<1$, the last equality is equivalent to (4) in the statement of the theorem.

Let us consider the case $\alpha>1$. For the segment $[0, \pi / 2-\delta]$ in a $u$-plane, there is a preimage $\left[\varepsilon_{1}, 1\right]$ in a $z$-plane and, consequently, a preimage $[\varepsilon, 1]$ in a $\zeta$-plane. Integrating (8) along these segments we obtain

$$
\varepsilon=\left(\frac{\alpha-1}{\alpha+1}\right)^{1 / \alpha} \frac{\delta}{2}+O\left(\delta^{2}\right)
$$

and finally, since $\delta=(c-1) / 2 c \varepsilon_{1}+O\left(\varepsilon_{1}^{2}\right)$, we obtain

$$
\left|A_{1}\right|=\frac{4 c}{1-c}\left(\frac{\alpha+1}{\alpha-1}\right)^{1 / \alpha} .
$$

Since $\alpha>1$, the last equality is equivalent to (4) in the statement of the theorem.

Now we prove (4). When $\alpha<1$ the segment $\left[0, \theta_{1}-\delta\right]$ in the domain $D_{1}^{u}$ has a preimage $\left[1,1 / \varepsilon_{1}\right]$ in a $z$-plane and, consequently, a preimage $[\varepsilon, 1]$ in a $\zeta$-plane. Let $z=f_{1}(\zeta)=B_{-1} / \zeta+B_{0}+B_{1} \zeta+\cdots$ be a function from $U$ onto $D_{1}^{*}$. From (6) one can derive

$$
\delta=\sqrt{c} \frac{1-c}{1+c} \varepsilon_{1}+O\left(\varepsilon_{1}^{2}\right) .
$$

Fixing the branch of the root in(12) with (+) after the equality sign and integrating (8) along the chosen segments we have

$$
\varepsilon=\left(\frac{1+\alpha}{1-\alpha}\right)^{\alpha} \frac{1+c}{4 \sqrt{c}} \delta+O\left(\delta^{2}\right)
$$

and finally using (12) we obtain

$$
\frac{1}{\left|B_{-1}\right|}=\frac{4}{1-\alpha^{2}}\left(\frac{1-\alpha}{1+\alpha}\right)^{\alpha}
$$

Since $\alpha<1$, the last equality is equivalent to (4) in the statement of the theorem. 
For $\alpha>1$, the segment $[\theta+\delta, \pi]$ in a $u$-plane has a preimage $\left[1 / \varepsilon_{1}, c\right]$ in a $z$-plane, and, consequently, a preimage $[\varepsilon, 1]$ in a $\zeta$-plane. Integrating $(8)$ along these segments we obtain

$$
\frac{1}{\left|B_{-1}\right|}=\frac{4}{\alpha^{2}-1}\left(\frac{\alpha-1}{\alpha+1}\right)^{\alpha}
$$

This is equivalent to (4) in the statement of the theorem. This completes the proof.

Similar calculation can also be found in [1].

Now we consider another modulus problem which is connected with the previous one. Let $S_{0}=\mathbb{C} \backslash\{0, c, 1\}$ be a punctured Riemanmsphere, $c \in(0,1)$. We consider a pair of curves $\left(\gamma_{1}, \gamma_{2}\right)$ on $S_{0}$, where $\gamma_{1}=\{w:|w|=1 / \varepsilon\}$ and $\gamma_{2}=\{w:|w|=\varepsilon\}$. Here $\varepsilon$ is sufficiently small and such that $c$ and 1 belong to the doubly connected domain between $\gamma_{1}$ and $\gamma_{2}$ on $\mathbb{C}$. Let $\mathfrak{B}$ be the set of all pairs $\left(B_{1}, B_{2}\right)$ consisting of circular domains of homotopy type $\left(\gamma_{1}, \gamma_{2}\right)$. Then the problem of the extremal partition of $S_{0}$ reduces to the problem of finding the maximum of the sum

$$
\alpha_{1}^{2} m\left(B_{1}, \infty\right)+\alpha_{2}^{2} m\left(B_{2}, 0\right),
$$

as $\left(B_{1}, B_{2}\right) \in \mathfrak{B}$. Without loss of generality we assume again that $\alpha_{1}=1, \alpha_{2}=\alpha$, $\alpha \in[0, \infty)$. We denote by $M(\alpha, c)$ the maximum of the sum (13). For $\alpha \leq \sqrt{c}$, this problem is equivalent to the previous one and $M(\alpha, c)=M(\alpha), \alpha<1$. For $\alpha \geq 1 / \sqrt{c}$, this problem is also equivalent to the previous one with $\alpha>1$ and $M(\alpha, c)=M(\alpha) / \sqrt{c}$.

From [8] it follows that there is a unique pair $\left(B_{1}^{*}, B_{2}^{*}\right)$ which is extremal for this problem. $B_{1}^{*}$ and $B_{2}^{*}$ are the circular domains in the trajectory structure of the differential $\Phi(z) d z^{2}=-A(z-b)^{2} d z^{2} / z^{2}(z-1)(z-c)$.

The following theorem is proved by analogy with the preceding one.

THEOREM 2.2. Let $\sqrt{c}<\alpha<1 / \sqrt{c}$. Then

$$
\begin{aligned}
& m\left(B_{1}^{*}, \infty\right)=\frac{1}{2 \pi} \log \frac{4(1-\sqrt{c})^{\alpha-1}}{(1+\sqrt{c})^{\alpha+1}}, \\
& m\left(B_{2}^{*}, 0\right)=\frac{1}{2 \pi} \log \frac{4 c(1-\sqrt{c})^{(1 / \alpha)-1)}}{(1+\sqrt{c})^{(1 / \alpha)+1}} .
\end{aligned}
$$

Moreover, for the differential $\Phi(z) d z^{2}$, we calculate $A=1 / 4 \pi^{2}$ and $b=\alpha \sqrt{c}$.

2.3. Let $U_{z}=U \backslash\{0, \omega\}, \omega \in(0,1)$ be the punctured unit disk. We consider the system of curves $\left(\gamma_{1}^{z}, \gamma_{2}^{z}\right)$ on $U_{2}$, where $\gamma_{2}^{z}=\{z:|z-\omega|=\varepsilon\}$ and $\gamma_{1}^{z}=\{z:|z|=\varepsilon\}$. Here $\varepsilon$ is sufficiently small and such that $\omega+\varepsilon<1$ and $\varepsilon<\omega / 2$. Let $\mathfrak{D}^{z}$ be the set of all pairs $\left(D_{1}^{z}, D_{2}^{z}\right)$ consisting of simply connected domains of homotopy type $\left(\gamma_{1}^{z}, \gamma_{2}^{z}\right)$. 
Then the problem of extremal partition of $U_{z}$ reduces to the problem of finding the maximum of the sum $m\left(D_{1}^{z}, 0\right)+\alpha^{2} m\left(D_{2}^{z}, \omega\right)$ as $\left(D_{1}^{z}, D_{2}^{z}\right) \in \mathfrak{D}^{z}$. This maximum is denoted by $M_{z}(\alpha, \omega)$. Under the transformation

$$
Z(z)=1-\frac{\omega(1+z)^{2}}{z(1+\omega)^{2}}
$$

two extremal domains $\left(D_{1}^{z^{*}}, D_{2}^{z^{*}}\right)$ in the problem for $M_{z}(\alpha, \omega)$ are mapped onto two extremal domains $\left(B_{1}{ }^{*}, B_{2}{ }^{*}\right)$ in the problem of finding $M(\alpha, c)$, where $c=$ $(1-\omega)^{2} /(1+\omega)^{2}$. Taking into account the change of reduced moduli under the conformal map $Z(z)$, we deduce that for $(1-\omega) /(1+\omega) \leq \alpha \leq(1+\omega) /(1-\omega)$,

$$
m\left(D_{1}^{z^{*}}, 0\right)=\frac{1}{2 \pi} \log \omega^{\alpha}, \quad m\left(D_{2}^{z^{*}}, \omega\right)=\frac{1}{2 \pi} \log \omega^{1 / \alpha}\left(1-\omega^{2}\right) .
$$

Let $U_{w}=U(M) \backslash\{0, \omega\}$ be the punctured disk of radius $M$. We consider on $U_{w}$ the system of curves $\left(\gamma_{1}^{w}, \gamma_{2}^{w}\right)$, where $\gamma_{2}^{w}=\{w:|w-\omega|=\varepsilon\}$ and $\gamma_{1}^{w}=\{w:|w|=\varepsilon\}$. Here $\varepsilon$ is sufficiently small. Let $\mathfrak{D}^{w}$ be the set of all pairs $\left(D_{1}^{w}, D_{2}^{w}\right)$ consisting of simply connected domains of homotopy type $\left(\gamma_{1}^{w}, \gamma_{2}^{w}\right)$. Then the problem of extremal partition of $U_{w}$ reduces to the problem of finding the maximum of the sum $m\left(D_{1}^{w}, 0\right)+\alpha^{2} m\left(D_{2}^{w}, \omega\right)$ as $\left(D_{1}^{w}, D_{2}^{w}\right) \in \mathfrak{D}^{z}$. This maximum is denoted by $M_{w}(\alpha, \omega)$. Theorem 2.1 and Theorem 2.2 and suitable conformal maps imply that

$$
\begin{aligned}
& m\left(D_{1}^{w *}, 0\right)= \begin{cases}\frac{1}{2 \pi} \log M^{1-\alpha} \omega^{\alpha}, & \text { for } \frac{M-\omega}{M+\omega} \leq \alpha \leq \frac{M+\omega}{M-\omega} \\
\frac{1}{2 \pi} \log \frac{M^{2} \omega}{(M+\omega)^{2}} \frac{4(1-\alpha)^{\alpha-1}}{(1+\alpha)^{\alpha+1}}, & \text { for } 0 \leq \alpha \leq \frac{M-\omega}{M+\omega} \\
\frac{1}{2 \pi} \log \frac{M^{2} \omega}{(M-\omega)^{2}} \frac{4(\alpha-1)^{\alpha-1}}{(\alpha+1)^{\alpha+1}}, & \text { for } \alpha \geq \frac{M+\omega}{M-\omega}\end{cases} \\
& m\left(D_{2}^{\omega *}, \omega\right)= \begin{cases}\frac{1}{2 \pi} \log \frac{\omega^{1 / \alpha}}{M^{1+\alpha}}\left(M^{2}-\omega^{2}\right), & \text { for } \frac{M-\omega}{M+\omega} \leq \alpha \leq \frac{M+\omega}{M-\omega} \\
\frac{1}{2 \pi} \log \frac{\omega(M+\omega)}{M-\omega} \frac{4 \alpha^{2}(1-\alpha)^{1 / \alpha-1}}{(1+\alpha)^{1 / \alpha+1}}, & \text { for } 0 \leq \alpha \leq \frac{M-\omega}{M+\omega} \\
\frac{1}{2 \pi} \log \frac{\omega(M-\omega)}{M+\omega} \frac{4 \alpha^{2}(\alpha-1)^{1 / \alpha-1}}{(\alpha+1)^{1 / \alpha+1}}, & \text { for } \alpha \geq \frac{M+\omega}{M-\omega}\end{cases}
\end{aligned}
$$

Here $\left(^{*}\right)$ denotes extremality of a domain.

2.4. We are concerned with a notion which appeared rather recently in $[2,9,13]$ and nowadays is used for extremal problems for conformal maps (see, for example, $[2,11,13,17])$. It is called the reduced modulus of a digon. We define the reduced moduli of digons following Emel'yanov [2], Kuz'mina [9], and Solynin [13]. 
Let $D$ be a hyperbolic simply connected domain from $\mathbb{C}$ with two finite fixed boundary points $a, b$ (maybe with the same support) on its boundary $\partial D$. We call it a digon. Let $S(a, \varepsilon)$ be a connected component of $D \cap\{|z-a|<\varepsilon\}$, such that $a \in \partial S(a, \varepsilon)$. We denote by $D_{\varepsilon}$ the domain $D \backslash\left\{S\left(a, \varepsilon_{1}\right) \cup S\left(b, \varepsilon_{2}\right)\right\}$ for sufficiently small $\varepsilon_{j}, j=1,2$. Let $M\left(D_{\varepsilon}\right)$ be a modulus of the family of arcs in $D_{\varepsilon}$ joining the boundary arcs of $S\left(a, \varepsilon_{1}\right)$ and $S\left(b, \varepsilon_{2}\right)$ that lie in the circumferences $|z-a|=\varepsilon_{1}$ and $|z-b|=\varepsilon_{2}$ (we choose a single arc in each circle so that both arcs can be connected in $D_{\varepsilon}$ ). If the limit

$$
m(D, a, b)=\lim _{\varepsilon_{1.2} \rightarrow 0}\left(\frac{1}{M\left(D_{\varepsilon}\right)}+\frac{1}{\varphi_{a}} \log \varepsilon_{1}+\frac{1}{\varphi_{b}} \log \varepsilon_{2}\right),
$$

exists, where $\varphi_{a}=\sup \Delta_{a}$ and $\varphi_{b}=\sup \Delta_{b}$ are the inner angles and $\Delta_{a}$ and $\Delta_{b}$ are the Stolz angles inscribed in $D$ at $a$ or $b$ respectively, then it is called the reduced modulus of a digon $D$. The existence of the limit is the local characteristic [13, Theorem 1.2] of the domain $D$. Suppose that there exists a conformal map $f(z)$ from the domain $S\left(a, \varepsilon_{1}\right) \subset D$ onto a circular sector, so that there exists the angular limit $f(a)$, which is the vertex of this sector and with the angle $\varphi_{a}$. If the function $f$ has the angular finite non-zero derivative $f^{\prime}(a)$, we say that the domain $D$ is conformal at the point $a$. If the digon $D$ is conformal at the points $a, b$, then the limit in the definition of $m(D, a, b)$ exists (see [13, Theorem 1.3]).

Suppose that there exists a conformal map $f(z)$ of the digon $D$ (which is conformal at $a, b$ ) onto a digon $D^{\prime}$, so that there exist the angular limits $f(a), f(b)$ with the inner angles $\psi_{a}$ and $\psi_{b}$ at the vertices $f(a)$ and $f(b)$ which are also thought of as the supremum over all Stolz angles inscribed in $D^{\prime}$ with the vertices $w_{1}=f(a)$ or $w_{2}=f(b)$ respectively. If the function $f$ has the angular finite non-zero derivatives $f^{\prime}(a)$ and $f^{\prime}(b)$, then $\varphi_{a}=\psi_{a}, \varphi_{b}=\psi_{b}$, and the reduced modulus exists and is changed by the rule

$$
m(f(D), f(a), f(b))=m(D, a, b)+\frac{1}{\psi_{a}} \log \left|f^{\prime}(a)\right|+\frac{1}{\psi_{b}} \log \left|f^{\prime}(b)\right| .
$$

If we suppose, moreover, that $f$ has the expansion

$$
f(z)=w_{1}+(z-a)^{\psi_{a} / \varphi_{a}}\left(c_{1}+c_{2}(z-a)+\cdots\right)
$$

about the point $a$, and the expansion

$$
f(z)=w_{2}+(z-b)^{\psi_{b} / \varphi_{b}}\left(d_{1}+d_{2}(z-a)+\cdots\right)
$$

about the point $b$, then the reduced modulus of $D$ is changed by the rule

$$
m(f(D), f(a), f(b))=m(D, a, b)+\frac{1}{\psi_{a}} \log \left|c_{1}\right|+\frac{1}{\psi_{b}} \log \left|d_{1}\right|,
$$


where $c_{1}, d_{1}$ are some complex non-zero constants. Obviously, one can extend this definition to the case of vertices with the infinite support.

Let us state now a problem about extremal partition of the disk $U(M)$. Let $0, \omega$ be punctures in $U(M)$. We consider the family $\mathbb{D}$ of digons $D$ in $U$ with the vertices $0, \omega \in \partial D$. We suppose that the inner angles of $D$ at these vertices are equal to $2 \pi$. The minimum $\min _{D \in \mathbf{D}} m(D, 0, \omega)$ is to be found. There is a unique digon $D_{1}^{*}=U(M) \backslash\{(-M, 0] \cup[\omega, M)\}$ that gives this minimum. Its reduced modulus is calculated by means of a suitable conformal map of $D^{*}$ onto the digon $\mathbb{C} \backslash[0, \infty)$ of modulus zero with respect to its vertices $0, \infty$. Thus we have

$$
m\left(D^{*}, 0, \omega\right)=\frac{1}{2 \pi} \log \frac{M^{2} \omega^{2}}{M^{2}-\omega^{2}} .
$$

3. Evaluation of the system of functionals $\left(\left|f^{\prime}(0)\right|,\left|f^{\prime}(\omega)\right|\right), f \in \mathscr{M}^{M}(\omega)$

In this section we study the mutual change of $\left|f^{\prime}(0)\right|$ and $\left|f^{\prime}(\omega)\right|$ over the class $f \in \mathscr{M}^{M}(\omega)$. Denote by $\Gamma^{+}$the arc of $y=\max \left|f^{\prime}(\omega)\right|$ as $f \in \mathscr{M}^{M}(\omega)$ and $\left|f^{\prime}(0)\right|=x$, and by $\Gamma^{-}$the arc of $y=\min \left|f^{\prime}(\omega)\right|$ as $f \in \mathscr{M}^{M}(\omega)$ and $\left|f^{\prime}(0)\right|=x$. We determine here $\mathfrak{M}_{f}$ by the moduli calculated in Section 2 . Let us set the functions

and

$$
g(z)=\frac{z}{1-u z+z^{2}}, \quad u \in[-2,2]
$$

$$
G(z, u)=M g^{-1}\left(M\left(\frac{1-u \omega+\omega^{2}}{M^{2}-M u \omega+\omega^{2}}\right) g(z)\right) .
$$

THEOREM 3.1. The boundary curve $\Gamma^{-}$of the range $\mathfrak{M}_{f}$ of the system of functionals $\left(\left|f^{\prime}(0)\right|,\left|f^{\prime}(\omega)\right|\right)$ in the class $\mathscr{M}^{M}(\omega)$ is given parameterically by $(x(u), y(u))$, $u \in[-2,2]$, where

$$
x=\frac{M^{2}\left(1-u \omega+\omega^{2}\right)}{M^{2}-M u \omega+\omega^{2}}, \quad y=\frac{\left(1-\omega^{2}\right)\left(M^{2}-M u \omega+\omega^{2}\right)}{\left(M^{2}-\omega^{2}\right)\left(1-u \omega+\omega^{2}\right)},
$$

as $-2 \leq u \leq 2$. The extremal function $G(z, u)$ is unique for each $u$.

Proof. Since

$$
\frac{M^{2}(1-\omega)^{2}}{(M-\omega)^{2}} \leq\left|G^{\prime}(0)\right|=\frac{M^{2}\left(1-u \omega+\omega^{2}\right)}{M^{2}-M u \omega+\omega^{2}} \leq \frac{M^{2}(1+\omega)^{2}}{(M+\omega)^{2}}
$$

for $u \in[-2,2]$, we choose for a function $f \in \mathscr{M}^{M}(\omega)$ the unique

$$
u=u_{0}=\frac{M^{2}(1-x)+\omega^{2}\left(M^{2}-x\right)}{M \omega(M-x)},
$$


so that $x=\left|f^{\prime}(0)\right|=G^{\prime}(0)$. Let us consider the digon $U_{z}^{\prime}=U \backslash\{(-1,0] \cup[\omega, 1)\}$ with two vertices $0, \omega$. Its reduced modulus is given as

$$
m\left(U_{z}^{\prime}, 0, \omega\right)=\frac{1}{2 \pi} \log \frac{\omega^{2}}{1-\omega^{2}} .
$$

The digon $f\left(U_{z}^{\prime}\right)$ with the vertices at $0, \omega$ has the reduced modulus

$$
m\left(f\left(U_{z}^{\prime}\right), 0, \omega\right)=\frac{1}{2 \pi} \log \frac{\omega^{2}}{1-\omega^{2}}\left|f^{\prime}(\omega)\right| x .
$$

It is admissible in the problem of minimum of the reduced modulus over all digons from the family $\mathbb{D}$. The extremal reduced modulus is

$$
m\left(D_{1}^{*}, 0, \omega\right)=\frac{1}{2 \pi} \log \frac{M^{2} \omega^{2}}{M^{2}-\omega^{2}},
$$

which is given by the digon obtained from $U(M) \backslash\{(-M, 0] \cup[\omega, M)\}$. Therefore,

$$
\left|f^{\prime}(\omega)\right| \geq \frac{M^{2}\left(1-\omega^{2}\right)}{\left(M^{2}-\omega^{2}\right) x} .
$$

The uniqueness of the extremal configuration $G\left(U_{z}^{\prime}\right)$ leads to the uniqueness of the extremal map.

We define now the curve $\Gamma^{+}$. For this we need the following technical lemma.

LEMMA 3.2. For $(1-\omega) /(1+\omega) \leq \alpha \leq(1+\omega) /(1-\omega)$, the function

$$
x(\alpha)=\exp \left(2 \pi\left(m\left(D_{1}^{w *}, 0\right)-m\left(D_{1}^{z *}, 0\right)\right)\right)
$$

is continuous and strictly decreases in $\alpha$

$$
\text { from } M^{2}(1+\omega)^{2} /(M+\omega)^{2} \text { to } M^{2}(1-\omega)^{2} /(M-\omega)^{2} \text {. }
$$

Here the quantities of $m\left(D_{1}^{u *}, 0\right)$ and $m\left(D_{1}^{z^{*}}, 0\right)$ are defined in Section 2.4.

Proof. For $\alpha \in[(M-\omega) /(M+\omega),(M+\omega) /(M-\omega)]$, the value of $x(\alpha)=M^{1-\alpha}$ decreases in $\alpha$. For $\alpha \in[(1-\omega) /(1+\omega),(M-\omega) /(M+\omega)]$, we have the derivative

$$
\frac{x^{\prime}(\alpha)}{x(\alpha)}=\log \frac{1-\alpha}{(1+\alpha) \omega}
$$

This implies that $x^{\prime}(\alpha)<0$. The case $\alpha \in[(M+\omega) /(M-\omega),(1+\omega) /(1-\omega)]$ is considered analogously. 
THEOREM 3.3. (i) The upper boundary curve $\Gamma^{+}$of the range $\mathfrak{M}_{f}$ of the system of functionals $\left(\left|f^{\prime}(0)\right|,\left|f^{\prime}(\omega)\right|\right)$ in the class $\mathscr{M}^{M}(\omega)$ consists of points $(x(\alpha), y(\alpha))$, $\alpha \in[(1-\omega) /(1+\omega),(1+\omega) /(1-\omega)]$, where

$$
x(\alpha)=\frac{4 M^{2} \omega^{1-\alpha}}{(M+\omega)^{2}} \frac{(1-\alpha)^{\alpha-1}}{(1+\alpha)^{\alpha+1}}, \quad y(\alpha)=\frac{4 \omega^{1-1 / \alpha}(M+\omega)}{\left(1-\omega^{2}\right)(M-\omega)} \frac{\alpha^{2}(1-\alpha)^{1 / \alpha-1}}{(1+\alpha)^{1 / \alpha+1}},
$$

for $\alpha \in[(1-\omega) /(1+\omega),(M-\omega) /(M+\omega)]$;

$$
x(\alpha)=M^{1-\alpha}, \quad y(\alpha)=\frac{M^{2}-\omega^{2}}{M^{1+1 / \alpha}\left(1-\omega^{2}\right)},
$$

for $\alpha \in[(M-\omega) /(M+\omega),(M+\omega) /(M-\omega)]$

$$
x(\alpha)=\frac{4 M^{2} \omega^{1-\alpha}}{(M-\omega)^{2}} \frac{(\alpha-1)^{\alpha-1}}{(\alpha+1)^{\alpha+1}}, \quad y(\alpha)=\frac{4 \omega^{1-1 / \alpha}(M-\omega)}{\left(1-\omega^{2}\right)(M+\omega)} \frac{\alpha^{2}(\alpha-1)^{1 / \alpha-1}}{(\alpha+1)^{1 / \alpha+1}},
$$

for $\alpha \in[(M+\omega) /(M-\omega),(1+\omega) /(1-\omega)]$.

(ii) Each point $(x(\alpha), y(\alpha))$ of $\Gamma^{+}$is attained by the unique function $F(z, \alpha)$ satisfying the differential equation $\varphi(z) d z^{2}=\psi(w) d w^{2}$, where

$$
\varphi(z) d z^{2}=\frac{(z-d)^{2}(z-\bar{d})^{2}}{z^{2}(z-\omega)^{2}(z-1 / \omega)^{2}} d z^{2}
$$

such that $|d|=1$ and $d$ is one of conjugated solutions of the equation

$$
\begin{gathered}
\alpha \frac{1-\omega}{1+\omega}=1-\frac{\omega(1+d)^{2}}{d(1+\omega)^{2}}, \\
\psi(w) d w^{2}=\frac{(w-c)\left(w-M^{2} / c\right)}{w^{2}(w-\omega)^{2}\left(w-M^{2} / \omega\right)^{2}} d w^{2}
\end{gathered}
$$

where $c=c(\alpha)$ is the unique solution in $(\omega, M)$ of the equation

$$
1-\alpha^{2}=\frac{\omega(M+c)^{2}}{c(M+\omega)^{2}} \quad \text { for } \alpha \in\left[\frac{1-\omega}{1+\omega}, \frac{M-\omega}{M+\omega}\right]
$$

$c=c(\alpha)$ is the unique solution in $(-M, 0)$ of the equation

$$
\begin{gathered}
1-\alpha^{2}=\frac{\omega(M-c)^{2}}{c(M-\omega)^{2}} \quad \text { for } \alpha \in\left[\frac{M+\omega}{M-\omega}, \frac{1+\omega}{1-\omega}\right] ; \\
\psi(w) d w^{2}=\frac{(w-c)^{2}\left(w-M^{2} / c\right)^{2}}{w^{2}(w-\omega)^{2}\left(w-M^{2} / \omega\right)^{2}} d w^{2},
\end{gathered}
$$

where $c=c(\alpha)$ is such that $|c|=M$ and $c$ is one of the solutions of the equation

$$
\alpha \frac{1-\omega}{1+\omega}=1-\frac{\omega(M+c)^{2}}{c(M+\omega)^{2}} \quad \text { for } \alpha \in\left[\frac{M+\omega}{M-\omega}, \frac{M+\omega}{M-\omega}\right] .
$$


The function $F(z, \alpha)$ maps the unit disk onto the disk $U(M)$ minus a piecewise analytic curve with two symmetric endpoints. The simply connected domain $F(U, \alpha)$ has the reduced modulus $x(\alpha)$ with respect to the origin.

Proof. Let $f \in \mathscr{M}^{M}(\omega)$ be a function with a fixed value of $\left|f^{\prime}(0)\right|=x$. The previous lemma asserts that there is a unique $\alpha$ such that $x(\alpha)=x$. Let us consider the functions $f_{1}(z)$ and $f_{2}(z)$ satisfying the equations

$$
\left(\frac{d f_{1}(z)}{f_{1}(z)}\right)^{2}=4 \pi^{2} \varphi(z) d z^{2}, \quad\left(\frac{d f_{2}(w)}{f_{2}(w)}\right)^{2}=4 \pi^{2} \psi(w) d w^{2},
$$

where the differentials $\varphi(z) d z^{2}$ and $\psi(w) d w^{2}$ are defined in the statement of the theorem and $\alpha$ is chosen. Theorem 2.1-Theorem 2.2, the transformation $Z(z)$ and a suitable map from the surface $U_{w}$ (see Section 2.3) yield that the superposition $f_{2}^{-1} \circ f_{1}(z)$ maps conformally the domain $D_{1}^{z^{*}}$ onto the domain $D_{1}^{w *}$ and the form of the differentials $\varphi$ and $\psi$ follows from that of the differentials $Q$ and $\Phi$. Continuing this map analytically onto $D_{2}^{z *}$ we obtain the function $F(z, \alpha)$ that maps the unit disk onto the domain which is admissible with respect to the differential $\psi(w) d w^{2}$. This function satisfies the equality $F^{\prime}(0, \alpha)=x(\alpha)$ and meets all conditions of the theorem. Since the pair $\left(D_{1}^{w *}, D_{2}^{w *}\right)$ is extremal in the family $\mathfrak{D}^{w}$ we have the following chain of inequalities

$$
\begin{aligned}
m(f & \left.\left(D_{1}^{z^{*}}\right), 0\right)+\alpha^{2} m\left(f\left(D_{2}^{z^{*}}\right), \omega\right) \\
& =m\left(D_{1}^{z^{*}}, 0\right)+\alpha^{2} m\left(D_{2}^{z^{*}}, \omega\right)+\frac{1}{2 \pi} \log x(\alpha)+\frac{\alpha^{2}}{2 \pi} \log \left|f^{\prime}(\omega)\right| \\
& \leq m\left(D_{1}^{w *}, 0\right)+\alpha^{2} m\left(D_{2}^{\omega *}, \omega\right) \\
& =m\left(D_{1}^{z^{*}}, 0\right)+\alpha^{2} m\left(D_{2}^{z^{*}}, \omega\right)+\frac{1}{2 \pi} \log x(\alpha)+\frac{\alpha^{2}}{2 \pi} \log \left|F^{\prime}(\omega, \alpha)\right| .
\end{aligned}
$$

Therefore, $\left|f^{\prime}(\omega)\right| \leq\left|F^{\prime}(\omega, \alpha)\right|=y(\alpha)$. The uniqueness of the extremal configuration implies the uniqueness of the extremal function.

REMARK. The range $\mathfrak{M}_{f}$ of the system of functionals $\left(\left|f^{\prime}(0)\right|,\left|f^{\prime}(\omega)\right|\right)$ is invariant under the transformation

$$
(x, y) \rightarrow\left(y \frac{M^{2}\left(1-\omega^{2}\right)}{M^{2}-\omega^{2}}, x \frac{M^{2}-\omega^{2}}{M^{2}\left(1-\omega^{2}\right)}\right) .
$$

This can be seen changing $\alpha \rightarrow 1 / \alpha$ in Theorem 3.1 and Theorem 3.3. 


\section{References}

[1] S. E. Demin, 'Isoperimetric distortion problem for univalent Montel functions', Sibirsk. Mat. Zh. 37 (1996), 108-116; English translation: Siberian Math. J. 37 (1996) 94-101.

[2] E. G. Emel'yanov, 'On extremal partitioning problems', Zap. Nauchn. Sem. Leningrad. Otdel. Mat. Inst. Steklov. (LOMI) 154 (1986), 76-89; English translation: J. Soviet Math. 43 (1988) 2558-2566.

[3] J. A. Jenkins, 'On the existence of certain general extremal metrics', Ann. of Math. (2) 66 (1957), $440-453$.

[4] J. Krzyż, 'On univalent functions with two preassigned values', Ann. Univ. Mariae CurieSklodowska Sect. A 15 (1961), 57-77.

[5] — - 'Some remarks concerning my paper: 'On univalent functions with two preassigned values' ', Ann. Univ. Mariae Curie-Sklodowska Sect. A 16 (1962), 129-136.

[6] _ - 'On the region of variability of the ratio $f\left(z_{1}\right) / f\left(z_{2}\right)$ within the class $S$ of univalent functions', Ann. Univ. Mariae Curie-Sklodowska Sect. A 17 (1963), 55-64.

[7] J. Krzyż and E. Złotkiewicz, 'Koebe sets for univalent functions with two preassigned values', Ann. Acad. Sci. Fenn. Ser. Al Math. 487 (1971), 1-12.

[8] G. V. Kuz'mina, Moduli of families of curves and quadratic differentials, Trudy Mat. Inst. Steklov. 139 (1980), English translation: Proc. Steklov Inst. Math. (Amer. Math. Soc., Providence, 1982).

[9] — - 'Extremal properties of quadratic differentials with strip domains in the structure of the trajectories', Zap. Nauchn. Sem. Leningrad. Otdel. Mat. Inst. Steklov. (LOMI) 154 (1986), 110-129; English translation: J. Soviet Math. 43 (1988) 2579-2591.

[10] R. J. Libera and E. Złotkiewicz, 'Bounded Montel univalent functions', Colloq. Math. 56 (1988), 169-177.

[11] D. Mejía, Ch. Pommerenke and A. Vasil'ev, 'Distortion theorems for hyperbolically convex functions', Complex Variables Theory Appl. 44 (2001), 117-130.

[12] M. O. Reade and E. Złotkiewicz, 'On values omitted by univalent functions with two pre-assigned values', Compositio Math. 24 (1972), 355-358.

[13] A. Yu. Solynin, 'Moduli and extremal metric problems', Algebra i Analiz 11 (1999), 3-86; English translation: St. Petersburg Math. J. 11 (2000), 1-65.

[14] A. Vasil'ev and P. Pronin, 'Distortion theorem for the univalent Montel functions', in: First Russian M. Souslin conference on Foundations of Math. and Function Theory, October 16-21 (Abstracts) (Saratov Pedagogical Institute, 1989) pp. 103-104.

[15] _ 'On some extremal problems for bounded univalent functions with the Montel normalization', in: Second Russian M. Souslin conference on Foundations of Math. and Function Theory, October 4-11 (Abstracts) (Saratov Pedagogical Institute, 1991) pp. 64-65.

[16] _ ' 'On some extremal proplems for bounded univalent function with Montel's normalization', Demonstratio Math. 26 (1993), 703-707.

[17] _ 'The range of a system of functionals for the Montel univalent functions', Bol. Soc. Mat. Mexicana (3) 6 (2000), 177-190.

Departamento de Matemática

Universidad Técnica Federico Santa María

Casilla 110-V

Valparaíso

Chile

e-mail: avassili@mat.utfsm.cl 
